\title{
TECHNIQUES OF PERTURBATION ANALYSIS
}

\author{
(Invited Paper) \\ Peter van DE Kamp \\ Sproul Observatory, Swarthmore College
}

\begin{abstract}
RESUMEN
Se presenta un resumen histórico de los análisis de perturbaciones estelares y en seguida, una discusión de la precisión alcanzable y las fuentes de los errores observacionales. Se ha encontrado un valor provisional para la densidad de masa de las compañeras astrométricas: $0.063 \pm 0.018$ (e.p.) masas solares por parsec cúbico.
\end{abstract}

\section{ABSTRACT}

\begin{abstract}
A historical summary of stellar-perturbation analyses is presented, followed by a discussion of the attainable accuracy and the sources of observational errors. A provisional value for the mass-density of astrometric companions is found to be $0.063 \pm 0.018$ (p.e.) solar masses per cubic parsec.
\end{abstract}

\section{INTRODUGTION}

If "multiple" in our colloquium title is defined as three or more, then the present report on perturbations refers to double and multiple stars. Limiting ourselves to astrometric studies, these are made with long-focus telescopes through (1) the study of the positions of the visible components on a background of stars (galaxies in the distant future perhaps) (van de Kamp 1967), (2) the relative positions of the visible components of a binary star, either observed visually or through the multiple exposure technique developed by Hertzsprung (1920) and continued by Strand and others. In the former case, orientation and scale are provided by the reference background, in the latter case, a trail is used to provide orientation while scale plays a negligible role for any separation below a minute of arc.

\section{PERTURBATION IN THE OBSERVED PATH OF A BINARY OR MULTIPLE STAR}

The classical discovery is by H. L. Alden, who in a premature attempt to determine the mass ratio of $\mathrm{BD}+66^{\circ} 34$ (Mlb 377) found a perturbation in the brighter component of this visual binary. This object was studied by John L. Hershey (1973) at the Sproul Observatory; the still unseen companion in this, new triple system, is most likely a late $\mathrm{M}$ dwarf star.

$\mathrm{BD}+6^{\circ} 938$, with a faint proper motion companion, $165^{\prime \prime}$ distant, has a perturbation pointing to a dwarf $M$ star (Lippincott 1973, Martin and Ianna 1975). Neither the companion of $\mathrm{BD}+66^{\circ} 34 \mathrm{~A}$, nor that of $\mathrm{BD}+6^{\circ} 938$, have yet been seen. To the best of my knowledge no other discoveries of this type have been made, but Dr. Strand may later amend this situation.

A case of special interest is the eclipsing variable VW Cephei, for which a perturbation was measured by John L. Hershey (1975), the third component was seen by Heintz near the predicted position.

\section{PERTURBATIONS FIRST OBTAINED FROM RELATIVE POSITIONS}

Perturbations have been measured in the paths of the components of other binaries but only after a perturbation had been observed in the relative orbit of the two components. I am referring to Zeta Cancri, Xi Ursae Majoris and Zeta Aquarii. The perturbation in Zeta Cancri AB-C observed as early as 1880 by Seeliger 1914), proves this object to be 
a quadruple system. The perturbation in $\mathrm{Xi}$ Ursae Majoris, discovered spectroscopically by Wright for the A-component, observed visually by Nörlund (1905) was beautifully confirmed by the multiple exposure technique of Hertzsprung (1920). The Bcomponent proved to have a spectroscopic companion, thus making Xi UMa also a quadruple system. Both Zeta Cancri and Xi UMa have been the subjects of later studies by Gasteyer (1954), and Heintz (1966) respectively. Most likely the unseen astrometric companion of Zeta $\mathrm{Cnc}$ is a white dwarf, that of $\mathrm{Xi}$ UMa $\mathrm{A}$ an $\mathrm{M}$ dwarf.

The perturbation in the visual binary Zeta Aquarii was discovered by Strand (1942), later studied by Franz (1958) and by Harrington (1968). The unseen companion is attached to the B-component and is probably an $\mathrm{M}$ dwarf.

\section{ATTAINABLE ACC.URACY}

This brief historical summary will now be followed by some remarks and comments on the photographic techniques currently employed (van de Kamp 1975). Long-focus telescopes are desirable for this work, permitting the discovery of small perturbations. For the Sproul refractor the scale value is $1 \mu=0$ ".019. Modern measuring machines have contributed appreciably to improved accuracy, not to mention vastly increased speed of measuring. The order of accuracy of the combination telescope, photographic plate and measuring machine is near the level of one micron and will now be further discussed.

\section{a) Relative Positions}

A multi-exposure plate containing 40 to 50 exposures yields a probable error of about $0^{\prime \prime} .006$ for the relative position of the two components. By taking 4 to 6 plates per season, an accuracy of $0 .{ }^{\prime \prime} 003$ or better may be reached. As to the error in visual measures, I am not qualified to pass judgment, but it must be kept in mind that visual measurements can be carried out down to much smaller separations than the photographic technique permits. The general rule for the latter has been that separations more than about 3 or 4 seconds of arc, i.e., well-separated images, yield reliable results. However, at Sproul we recently have become aware of systematic effects in the measurements of component images, well- separated up to about six seconds of arc but apparently influencing each other. Systematic errors definitely appear for images which are close, in contact or are underexposed.

is $y, y$.

\section{b) Positions on background}

The positions in the path of a star, based on 2 to 4 exposures referred to a background of several reference stars may be obtained with an accuracy expressed by a probable error of one micron or about 0 ".02. By taking several plates on any one night this error may be reduced to 0.01 . By taking say 25 plates annually, a reduction to 0.002 would seem possible, except for the law of diminishing returns, resulting from a "year" error of about 0"002 (p.e.). At this moment we shall accept for the limiting accuracy obtainable for a normal point, based on an annual set of observations, a value of 0.003 , the same as attained in the multiple exposure technique.

There are of course the potential, and potentially appreciable errors, sometimes color-dependent and extending over many years, probably caused by changes in the optical adjustment of the telescope. These systematic errors are a subject of continued study with the Sproul refractor. Besides optical adjustment, ventilation in the dome may play a role. It is a source of some satisfaction that since the major change, namely to a new close-fitting cell for the objective in 1949, the optical performance of the Sproul refractor appears to have been rather stable.

Long-term intensive observations are required for this problem, as well as continued controls. In this, as in other cases, continued vigilance is the price of accuracy.

\section{GENERAL REMARKS. MASS DENSITY}

High accuracy, i.e. the reduction of the size of errors, both accidental and systematic is an obvious requirement in the search and study of perturbations. Equally important is the spread of observational material over long intervals of time. Extended, intensive, series of observations are a sine qua non, the only way to do justice to this problem, which often deals with perturbations of small amplitude, well below 0 ".05, and generally having periods of the order of one decade or more. A few perturbations 
with periods less than one decade have been found with correspondingly small amplitudes. - At present about fifteen perturbations with total amplitudes over 0".05 are known (van de Kamp 1975).

A statistical interpretation of the observed data for 19 well-established unseen astrometric companions yields a provisional value of $0.063 \pm 0.018$ (p.e.) $M_{\odot}$ per cubic parsec, a reasonable value considering results obtained from dynamical considerations and from visible stars. Further details may be found in Mass-Density of Unseen Astrometric Companions, published in the Proceedings of the Tercentenary Symposium on The Galaxy and the Local Group held at Herstmonceux Castle in July 1975.

\section{REFERENCES}

Franz, O. 1958, A.J. 63, 329.

Gasteyer, C. 1954, A.J., 59, 243.

Harrington, R. S. 1968, A.J., 73, 508.

Heintz, W. D. 1966, Veröff. Sternw. München, 7, 35.

Hershey, J.L. 1973, A.J., 78, 935.

Hershey, J. L. 1975, A.J., 80, 662.

Hertzsprung, E. 1920, Pub. Astrophys. Obs. Potsdam, Vol. $24, \mathrm{~N}^{0} 75$.

Lippincott, S. L. 1973, A.J., 78, 303.

Martin, G.E., and Ianna, P.A. 1975, A.J., 80, 321.

Nörlund, N.E. 1905, Astr. Nachr., 170, 9

Seeliger, H. 1914, Astr. Nachr., 199, 273.

Strand, K.Aa. 1942, A.J., 49, 165.

van de Kamp, P. 1967, Principles of Astrometry (San Francisco: Freeman).

van de Kamp, P. 1975, Ann. Rev. Astr. and Ap., 13, 295.

\section{DISCUSSION}

Poveda: I am worried about the mass per cubic parsec that you find for the unseen companions. When you consider that each unseen companion is substantially less massive than its primary and that not all primaries have companions, one should conclude that the mass of the unseen companions per cubic parsec should be substantially smaller than the mass of known ("visible") stars.

van de Kamp: The 19 unseen companions have an average (estimated) mass of $0.30 \mathcal{M}_{\odot}$. Their primaries have an average mass of $0.76 \mathcal{M}_{\odot}$. One could therefore conclude on a ratio of 2.5 to 1 for the mass density of visible relative to unseen stars.

In my presentation I have compared the mass density of the unseen companions with an independent determination of the mass density of seen stars, in which case near equality is indicated. In either case selection effects play a significant role. 